\title{
The effect of COVID-19 on emergency department imaging: what can we learn?
}

\author{
Camara Sharperson ${ }^{1} \cdot$ Tarek N. Hanna $^{2,3}$ (D) Keith D. Herr ${ }^{2} \cdot$ Matthew E. Zygmont $^{2} \cdot$ Roger L. Gerard $^{1} \cdot$ \\ Jamlik-Omari Johnson ${ }^{2}$
}

Received: 2 November 2020 / Accepted: 14 December 2020 / Published online: 8 January 2021

(C) American Society of Emergency Radiology 2021

\begin{abstract}
Purpose To investigate the effect of the COVID-19 pandemic on emergency department (ED) imaging.

Methods This retrospective study included all ED visits at a four-hospital academic health system in two matched 5-week periods. Demographic information, COVID-19 status, and disposition were reviewed. Type of imaging, acquisition time, and radiology reports were analyzed. Significance level was set at $p<0.05$.

Results A $43.2 \%$ decrease in ED visits and $12 \%$ reduction in overall ED imaging occurred during the pandemic period. Mean age was unchanged, but a shift in gender and racial characteristics was observed $(p<0.001)$. In the pandemic period, COVID-19 ED patients were older $(61.8 \pm 16.9$ years, $p<0.001)$ and more likely to be Black $(64.2 \% ; p<0.001)$ than non-COVID-19 patients. Imaging per ED encounter increased to $2.4 \pm 2.8$ exams from $1.7 \pm 1.1(p<0.001)$. Radiography increased $(57.2 \%$ vs. $52.4 \%)$ as a fraction of total ED imaging, while computed tomography (23.4\% vs. $27.2 \%)$ and ultrasound (8.5\% vs. $9.6 \%$ ) decreased (prepandemic vs. pandemic). COVID-19 ED patients underwent CT and US at a lower rate (11.5\% and 5.4\%) than non-COVID-19 patients $(25.4 \%$ and $9.1 \%)$. The proportion of imaging study reports concluding "no disease" or "no acute disease" decreased from 56.7 to $40.6 \%(p<0.001)$.

Conclusion The COVID-19 pandemic led to a significant reduction in ED visits, a shift in patient demographics, and a significant decrease in imaging volume. Additional impact included a significant increase in the proportion of positive imaging studies.
\end{abstract}

Keywords COVID-19 $\cdot$ Emergency Department $\cdot$ imaging $\cdot$ SARS-CoV-2 $\cdot$ radiology

\section{Introduction}

Published data have demonstrated that the SARS-CoV-2 (COVID-19) pandemic has affected the US healthcare system in unique respects. Infection control measures, such as social distancing, lockdowns, the use of telemedicine, and modifications in clinical inpatient as well as outpatient services, were

Tarek N. Hanna

tarek.hanna@emory.edu

1 Emory University School of Medicine, 1364 Clifton Road NE, Atlanta, GA 30322, USA

2 Department of Radiology and Imaging Sciences, Emory University School of Medicine, 1364 Clifton Road NE, Atlanta, GA 30322, USA

3 Division of Emergency and Trauma Imaging, Department of Radiology and Imaging Sciences, Emory University Midtown Hospital, 550 Peachtree Road, Atlanta, GA 30308, USA implemented across the country [1]. Moreover, patients have been encouraged to avoid hospitals except for true emergencies $[2,3]$ and to use telephone hotlines for symptom screening instead of presenting for in-person evaluation [3]. To optimize infection control and conserve healthcare resources, evaluation and treatment of chronic ailments were de-prioritized, potentially elevating morbidity and mortality from these underlying conditions due to delayed care [4-7]. Likewise, radiology departments postponed nonurgent imaging examinations and procedures [8].

During the early pandemic period (defined by Hartnett et al. as March 29-April 25), the total number of ED visits in the USA was $42 \%$ lower than the same period a year earlier [9], an observation attributed to patient concern of contracting COVID-19 from other patients in the ED [10, 11]. Lange et al. showed that, in the 10 weeks following the declaration of the pandemic in the USA, ED visits declined 23\% for myocardial infarction, $20 \%$ for stroke, and $10 \%$ for hyperglycemic crisis [12]. Similarly, Kim et al. reported that ED visits for 
conditions that would ordinarily warrant surgical consultation, such as appendicitis and bowel obstruction, decreased by $33.7 \%$, a trend that paralleled reductions in all major diagnoses in that study [11].

The purpose of this retrospective observational study was to investigate the effect of the COVID-19 pandemic on both COVID-19-related and non-COVID-19-related ED visits and associated ED imaging. Additionally, this study sought to determine whether the pandemic impacted the type of pathology diagnosed on imaging. Examining specific changes in ED patient presentation, imaging utilization and imaging pathology may help further elucidate the effect of the pandemic on population health.

\section{Materials and methods}

This retrospective cohort study on ED visits was conducted at a four-hospital healthcare system in a major metropolitan city in the southeastern US. The local institutional review board approved this study.

\section{Data source}

This healthcare network included a suburban academic quaternary care center with an American College of Surgery Level-2 trauma designation, approximately 40,000 annual ED visits, and 587 hospital beds; an urban academic hospital with a Level-2 trauma designation, approximately 65,000 annual ED visits, and 531 hospital beds; and two suburban community hospitals with Level-2 trauma designation, each with approximately 40,000 annual ED visits with 410 and 110 hospital beds respectively. Data were extracted from the clinical data warehouse.

\section{Study population and period}

March 23, 2020, was identified as the beginning of the pandemic period for this study, during which aspects of social life and healthcare operations were significantly impacted by a statewide executive order restricting certain social interactions to reduce transmission of the SARS-CoV-2 virus. Specifically, the order banned gatherings of over ten people, closed bars and nightclubs, and mandated that medically fragile individuals shelter-in-place [13]. A separate citywide stayat-home order was signed that directed all local residents to remain in their homes except when needing to perform essential tasks [14]. On April 27, 2020, stay-at-home restrictions were modified to allow the opening of most businesses in the state [15]. Therefore, the timeframe spanning March 23 and April 27 reflects a unique period to examine the impact of the most austere social distancing restrictions on healthcareseeking behavior and imaging ordering practices in the ED in the study region. All patients with ED visits within the healthcare network between March 23, 2020, and April 27, 2020 , were extracted from the enterprise-wide clinical data warehouse and categorized as the pandemic period cohort. All ED visits of the corresponding days of the week from the previous year (March 25, 2019, to April 27, 2019) were extracted as the pre-pandemic period comparator. Visits involving patients under 18 years were excluded from analysis.

Pandemic period patients were further divided into cohorts based on COVID-19 status. COVID-19 patients had a labconfirmed COVID-19 diagnosis during an ED encounter. A patient was classified as a "person under investigation" (PUI) if (1) PUI for COVID-19 was provided as an ED imaging study indication, or (2) COVID-19 was listed as an ED discharge diagnosis, but a laboratory test for COVID-19 was either negative or not performed during the ED encounter. Note that during this period PUI was an official designation in our healthcare system, used to ensure infection control compliance among staff and patients, and we believe a PUI status designation resulted in greater adherence than typical imaging study indications. All other patients who did not meet criteria as COVID-19 patients or PUIs were classified as nonCOVID-19 patients.

\section{Variables}

Demographic characteristics, including age, sex, race/ethnicity, and expected primary payer, were extracted. Clinical characteristics, including time of arrival to ED, arrival mode (ambulance, walk-in, private vehicle), length of stay (minutes), discharge status, ED disposition (admission, discharge, transfer), visit severity level (1-5), final ED diagnosis, and COVID-19 status (COVID-19, PUI or non-COVID-19). Imaging data were obtained for those patients who received ED imaging, to include type of imaging, time of first imaging examination, number of examinations, and all radiology reports. Natural language processing was used to determine positivity at imaging presentation with regard to the impression section of the radiology report. Imaging was considered normal if "no acute" or "normal" was in the impression section of the radiology report. The phrase "no acute" and the word "normal" were chosen as they appear in the standard departmental report templates, which have 99\% compliance for use among emergency radiologists at our institution.

\section{Statistical analysis}

Descriptive analyses were conducted to compare patient demographics and baseline clinical characteristics between cohorts. Frequencies and proportions were used to summarize categorical variables, and mean, standard deviation, and median were reported for continuous variables. Comparisons between cohorts were made using $t$ test for continuous variables 
and chi-square test for categorical variables. Statistical significance was defined as $\alpha=0.05$. All analyses were performed using SAS 9.4 (SAS Institute Inc, Cary, NC).

\section{Results}

\section{ED visits}

There were 11,108 ED visits during the pandemic period and 19,558 visits during the pre-pandemic period, indicating a $43.2 \%$ reduction in ED visits. The mean (SD) age of patients across both cohorts was similar: 50.9 (19.0) years for pandemic period patients and 51.2 (20.2) years for pre-pandemic period patients $(p=0.278)$. Demographics and clinical characteristics of patient by time period and COVID-19 status are presented in Table 1.

\section{Imaging}

There was a $12 \%$ reduction in ED imaging when comparing the pre-pandemic period to the pandemic period (22,172 prepandemic period examinations vs. 19,555 pandemic period examinations) (Table 2). Of note, however, a greater proportion of radiographs was ordered during the pandemic period (52.4\% vs. $57.2 \%, p<0.001)$ (Fig. 1). Among subtypes of radiographs, chest radiographs were performed significantly more during the pandemic period $(65.6 \%$ vs. $72.0 \%, p<$ 0.001 ) (Table 2). While the proportion of imaging that was CT decreased during the pandemic period (27.2\% vs. $23.4 \%$, $p<0.001$ ), of the CT examinations performed, chest CT increased significantly $(17.2 \%$ vs. $23.3 \%, p<0.001)$. Head CT significantly decreased $(35.6 \%$ vs. $32.5 \%, p<0.001)$.

While a reduction in the overall number of imaging studies was observed, the proportion of studies that were normal significantly decreased (56.7 to $40.6 \%, p<0.001)$ between cohorts (Table 2). Conversely, the mean (SD) number of imaging exams per encounter was significantly higher during the pandemic period [1.7 (1.1) vs. $2.4(2.8), p<0.001]$.

\section{Subgroup analysis}

During the pandemic period, there were 508 COVID-19 encounters, 1213 encounters involving PUIs, and 9387 ED visits that were not related to COVID-19 (Table 1). A majority of COVID-19 patients were male $(51.6 \%, p<0.001)$ and Black $(64.2 \%, p<0.001)$. COVID-19 patients were of a higher ED acuity status compared to PUIs and non-COVID-19 patients. Moreover, $79.7 \%$ of COVID-19 patients were admitted from the ED compared to $45.3 \%$ of PUIs and $25.4 \%$ of nonCOVID-19 patients $(p<0.001)$. There were 2011 imaging examinations performed on COVID-19 patients and on average (SD) there were 4.0 (5.1) exams per COVID-19-related encounter (Table 2). PUIs underwent an average (SD) of 2.8 (3.3) examinations per encounter, including CT at a greater frequency than COVID-19 patients $(21.8 \%$ vs. $11.5 \%)$ but fewer radiographs $(62.0 \%$ vs. $81.1 \%)$.

\section{Discussion}

Our data demonstrated that, in the early period of the COVID19 pandemic, significant differences in ED patient volume, demographic characteristics of patients presenting to EDs, management of ED patients, and ED imaging ordering patterns were observed compared to a similar period in the previous year (pre-pandemic). The overall decrease in ED patient visits (43.2\%, compared to 2019) observed in our study was in keeping with reports of significant reductions in ED patient volume and community emergency response activation calls in the USA described by others $[9,16]$. Hartnett et al. reported a nationwide decrease in ED patient visits by $42 \%$ in the latter part of our study period (April 12-18, 2020) compared to the prior year [9]. Preliminary data from another observational study reflected a $26.1 \%$ reduction in the number of Emergency Medical Services System (9-1-1) calls in the USA [16]. Garcia et al. observed a $38 \%$ reduction in STelevation myocardial infarctions (STEMI) activations across nine high-volume cardiac catheterization facilities in the USA [17]. A similar phenomenon has been reported for stroke outside the USA. In a study of 280 stroke centers out of China, a $40 \%$ reduction in stroke admissions was reported [18]. The authors of these studies postulated that decreased emergent healthcare utilization during the pandemic may have been attributable to a common fear among the general public of contracting COVID-19 in medical centers, a concern that in the USA was substantiated by public health communications that discouraged individuals from seeking ED care unless severely ill [11]. We speculate that our observation of a marked reduction in ED patient volumes can likewise be explained by the fear of contagion and widespread public health and news media messaging that all but the most severely ill patients should not seek in-person emergent medical care. Decreased ED visits could additionally have been due to a decrease in hazardous activity during lockdown, resulting in a reduction in minor trauma and subsequent injury-related ED visits.

Those who did present to the ED in our study period had different demographic characteristics when compared to the pre-pandemic period. While a majority of patients in both periods were female, the proportion of male patients significantly increased during the pandemic. Moreover, patients presenting to the ED were more likely to be Black and less likely to be Caucasian. Of those patients who were diagnosed with COVID-19 during their ED visit, $51.6 \%$ were male, and nearly two-thirds were Black and 


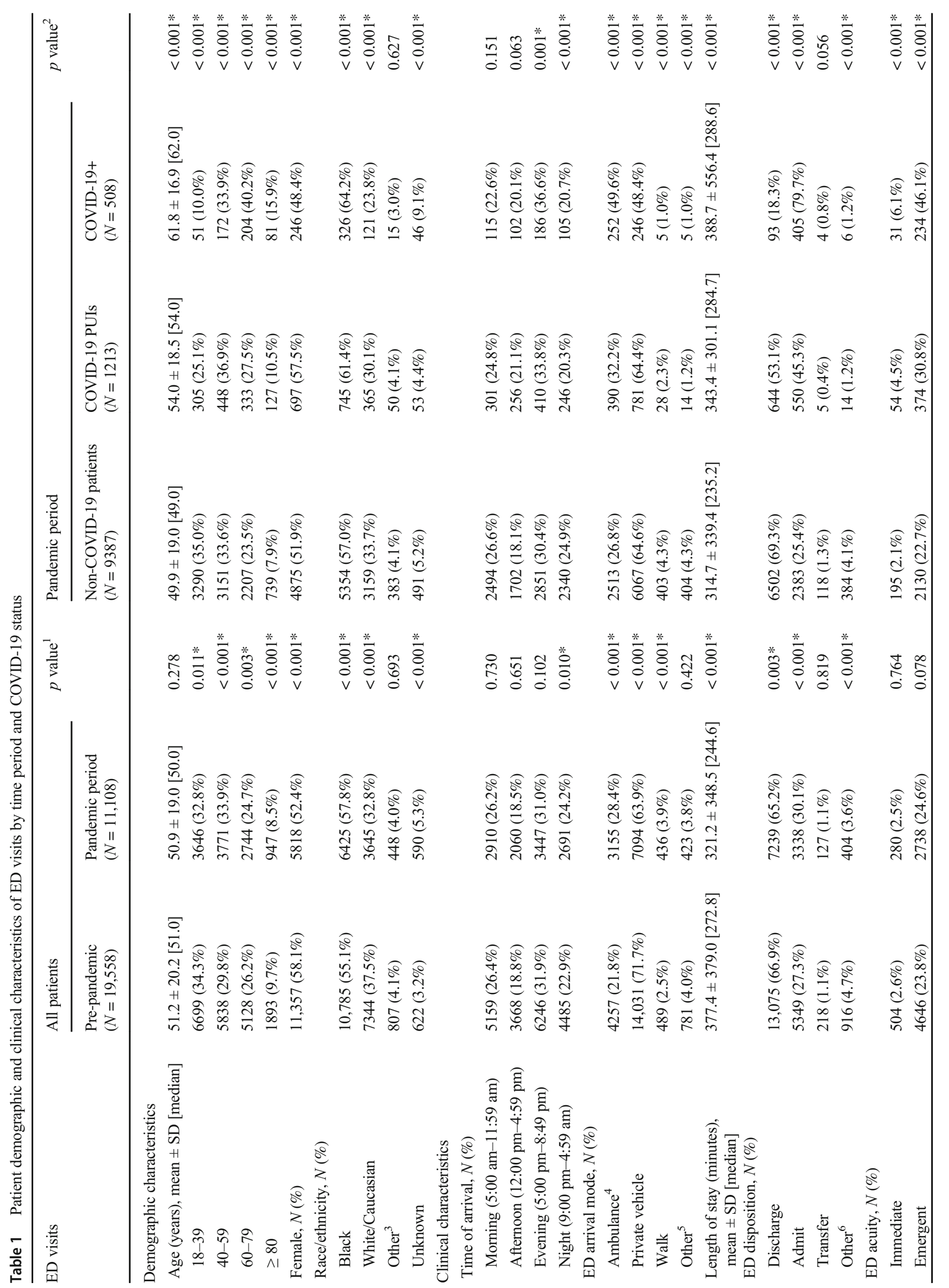




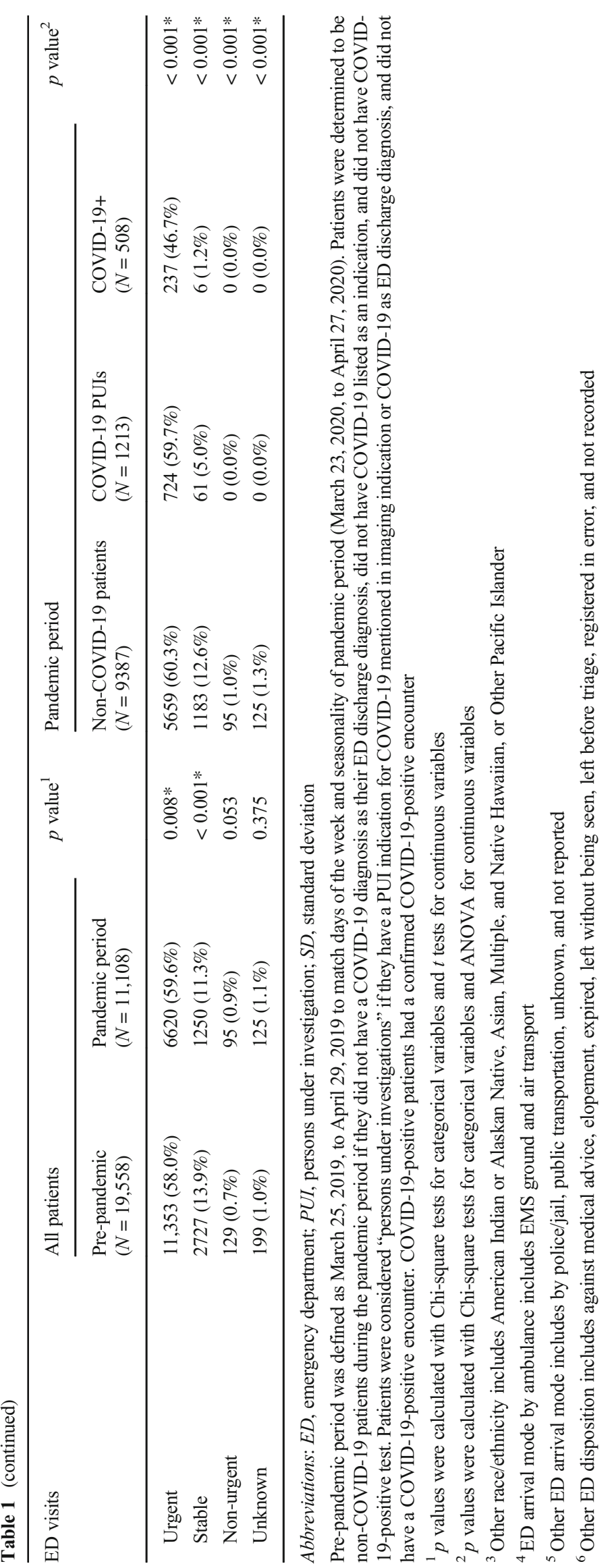


Table 2 Imaging performed for ED visits by time period and COVID-19 status

\begin{tabular}{|c|c|c|c|c|c|c|c|}
\hline \multirow[t]{2}{*}{ Imaging characteristics } & \multicolumn{2}{|l|}{ All patients } & \multirow[t]{2}{*}{$p$ value $^{1}$} & \multicolumn{3}{|c|}{ Patients during pandemic period } & \multirow[t]{2}{*}{$p$ value $^{2}$} \\
\hline & $\begin{array}{l}\text { Pre-pandemic } \\
\text { period } \\
(N=22,172)\end{array}$ & $\begin{array}{l}\text { Pandemic } \\
\text { period } \\
(N=19,555)\end{array}$ & & $\begin{array}{l}\text { Non-COVID-19 } \\
\text { patients } \\
(N=14,184)\end{array}$ & $\begin{array}{l}\text { COVID-19 PUIs } \\
(N=3360)\end{array}$ & $\begin{array}{l}\text { COVID-19+ } \\
(N=2011)\end{array}$ & \\
\hline \multicolumn{8}{|l|}{ Image modality, $N(\%)$} \\
\hline Radiograph & $11,612(52.4 \%)$ & $11,191(57.2 \%)$ & $<0.001 *$ & $7478(52.7 \%)$ & $2083(62.0 \%)$ & $1,630(81.1 \%)$ & $<0.001 *$ \\
\hline Chest & $7616(65.6 \%)$ & $8060(72.0 \%)$ & $<0.001 *$ & $5102(68.2 \%)$ & $1722(82.7 \%)$ & $1236(75.8 \%)$ & $<0.001 *$ \\
\hline Spine & $616(5.3 \%)$ & $197(1.8 \%)$ & $<0.001 *$ & $184(2.5 \%)$ & $13(0.6 \%)$ & $0(0.0 \%)$ & $<0.001 *$ \\
\hline Knee & $443(3.8 \%)$ & $164(1.5 \%)$ & $<0.001 *$ & $152(2.0 \%)$ & $7(0.3 \%)$ & $5(0.3 \%)$ & $<0.001 *$ \\
\hline Thoracoabdomen & $37(0.3 \%)$ & $679(6.1 \%)$ & $<0.001 *$ & $235(3.1 \%)$ & $139(6.7 \%)$ & $305(18.7 \%)$ & $<0.001 *$ \\
\hline Other & $2487(21.4 \%)$ & $1461(13.1 \%)$ & $<0.001 *$ & $1336(17.9 \%)$ & $111(5.3 \%)$ & $14(0.9 \%)$ & $<0.001 *$ \\
\hline Computed tomography $(\mathrm{CT})$ & $6032(27.2 \%)$ & $4569(23.4 \%)$ & $<0.001 *$ & $3606(25.4 \%)$ & $731(21.8 \%)$ & $232(11.5 \%)$ & $<0.001 *$ \\
\hline Head & $2148(35.6 \%)$ & $1485(32.5 \%)$ & $<0.001 *$ & $1250(34.7 \%)$ & $157(21.5 \%)$ & $78(33.6 \%)$ & $<0.001 *$ \\
\hline Abdomen and pelvis & $2027(33.6 \%)$ & $1422(31.1 \%)$ & $0.007^{*}$ & $1199(33.3 \%)$ & $178(24.4 \%)$ & $45(19.4 \%)$ & $<0.001 *$ \\
\hline Chest & $1037(17.2 \%)$ & $1066(23.3 \%)$ & $<0.001 *$ & $631(17.5 \%)$ & $337(46.1 \%)$ & $98(42.2 \%)$ & $<0.001 *$ \\
\hline Spine & $346(5.7 \%)$ & $215(4.7 \%)$ & $0.019^{*}$ & $193(5.4 \%)$ & $17(2.3 \%)$ & $5(2.2 \%)$ & $<0.001^{*}$ \\
\hline Other & $474(7.9 \%)$ & $381(8.3 \%)$ & 0.368 & $333(9.2 \%)$ & $42(5.7 \%)$ & $6(2.6 \%)$ & $<0.001 *$ \\
\hline Ultrasound & $2139(9.6 \%)$ & $1662(8.5 \%)$ & $<0.001 *$ & $1294(9.1 \%)$ & $259(7.7 \%)$ & $109(5.4 \%)$ & $<0.001 *$ \\
\hline Pelvis & $724(33.8 \%)$ & $276(16.6 \%)$ & $<0.001 *$ & $272(21.0 \%)$ & $4(1.5 \%)$ & $0(0.0 \%)$ & $<0.001 *$ \\
\hline Lower extremity & $504(23.6 \%)$ & $453(27.3 \%)$ & $0.009^{*}$ & $295(22.8 \%)$ & $100(38.6 \%)$ & $58(53.2 \%)$ & $<0.001 *$ \\
\hline Abdomen & $335(15.7 \%)$ & $338(20.3 \%)$ & $<0.001 *$ & $261(20.2 \%)$ & $58(22.4 \%)$ & $19(17.4 \%)$ & $0.003 *$ \\
\hline Retroperitoneum & $144(6.7 \%)$ & $228(13.7 \%)$ & $<0.001 *$ & $160(12.4 \%)$ & $52(20.1 \%)$ & $16(14.7 \%)$ & $<0.001 *$ \\
\hline Fetus & $163(7.6 \%)$ & $78(4.7 \%)$ & $<0.001 *$ & $74(5.7 \%)$ & $3(1.2 \%)$ & $1(0.9 \%)$ & $0.001 *$ \\
\hline Upper extremity & $78(3.6 \%)$ & $97(5.8 \%)$ & $0.001 *$ & $63(4.9 \%)$ & $20(7.7 \%)$ & $14(12.8 \%)$ & $<0.001 *$ \\
\hline Other & $191(8.9 \%)$ & $192(11.6 \%)$ & $0.008^{*}$ & $169(13.1 \%)$ & $22(8.5 \%)$ & $1(0.9 \%)$ & $<0.001 *$ \\
\hline $\begin{array}{l}\text { Magnetic resonance } \\
\text { imaging (MRI) }\end{array}$ & $658(3.0 \%)$ & $750(3.8 \%)$ & $<0.001^{*}$ & $652(4.6 \%)$ & $90(2.7 \%)$ & $8(0.4 \%)$ & $<0.001 *$ \\
\hline $\begin{array}{l}\text { Computed tomography } \\
\text { angiography (CTA) }\end{array}$ & $477(2.2 \%)$ & $490(2.5 \%)$ & $0.016^{*}$ & $437(3.1 \%)$ & $43(1.3 \%)$ & $10(0.5 \%)$ & $<0.001^{*}$ \\
\hline $\begin{array}{l}\text { Positron emission } \\
\text { tomography (PET) }\end{array}$ & $403(1.8 \%)$ & $222(1.1 \%)$ & $<0.001 *$ & $189(1.3 \%)$ & $33(1.0 \%)$ & $0(0.0 \%)$ & $<0.001 *$ \\
\hline $\begin{array}{l}\text { Magnetic resonance } \\
\text { angiography (MRA) }\end{array}$ & $329(1.5 \%)$ & $189(1.0 \%)$ & $<0.001^{*}$ & $172(1.2 \%)$ & $12(0.4 \%)$ & $5(0.2 \%)$ & $<0.001 *$ \\
\hline Other3 & $522(2.4 \%)$ & $482(2.5 \%)$ & 0.462 & $356(2.5 \%)$ & $109(3.2 \%)$ & $17(0.8 \%)$ & $<0.001 *$ \\
\hline $\begin{array}{l}\text { Number of images per } \\
\quad \text { encounter, mean } \pm \text { SD [median] }\end{array}$ & $1.7 \pm 1.1[1.0]$ & $2.4 \pm 2.8[1.0]$ & $<0.001 *$ & $2.2 \pm 2.3[1.0]$ & $2.8 \pm 3.3[2.0]$ & $4.0 \pm 5.1[2.0]$ & $<0.001 *$ \\
\hline Normal/non-acute images, $N(\%)$ & $12,581(56.7 \%)$ & $7,948(40.6 \%)$ & $<0.001 *$ & $6,544(46.1 \%)$ & $1,150(34.2 \%)$ & $254(12.6 \%)$ & $<0.001 *$ \\
\hline
\end{tabular}

Abbreviations: ED, emergency department; $P U I$, persons under investigation; $S D$, standard deviation

Pre-pandemic period was defined as March 25, 2019, to April 29, 2019, to match days of the week and seasonality of pandemic period (March 23, 2020, to April 27, 2020). Patients were determined to be non-COVID-19 patients during the pandemic period if they did not have a COVID-19 diagnosis as their ED discharge diagnosis, did not have COVID-19 listed as an indication, and did not have COVID-19 positive test. Patients were considered "persons under investigations" if they have a PUI indication for COVID-19 mentioned in imaging indication or COVID-19 as ED discharge diagnosis, and did not have a COVID-19-positive encounter. COVID-19-positive patients had a confirmed COVID-19-positive encounter

${ }^{1} p$ values were calculated with Chi-square tests for categorical variables and $t$ tests for continuous variables

${ }^{2} p$ values were calculated with Chi-square tests for categorical variables and ANOVA for continuous variables

${ }^{3}$ Other includes mammogram, interventional radiology, magnetic resonance venography, nuclear medicine, myocardial perfusion stress tests, and consultations from outside scans

over 60 years. Our findings are consistent with reported predilection of COVID-19 for Black, male, and older patients reported in the literature [19].
While ED patients overall were more likely to be admitted during the pandemic, nearly $80 \%$ of patients receiving a COVID-19 diagnosis, in particular, were ultimately admitted 
Fig. 1 Imaging by time period. Stacked bar charts summarizing the frequency of types of ED imaging by time period

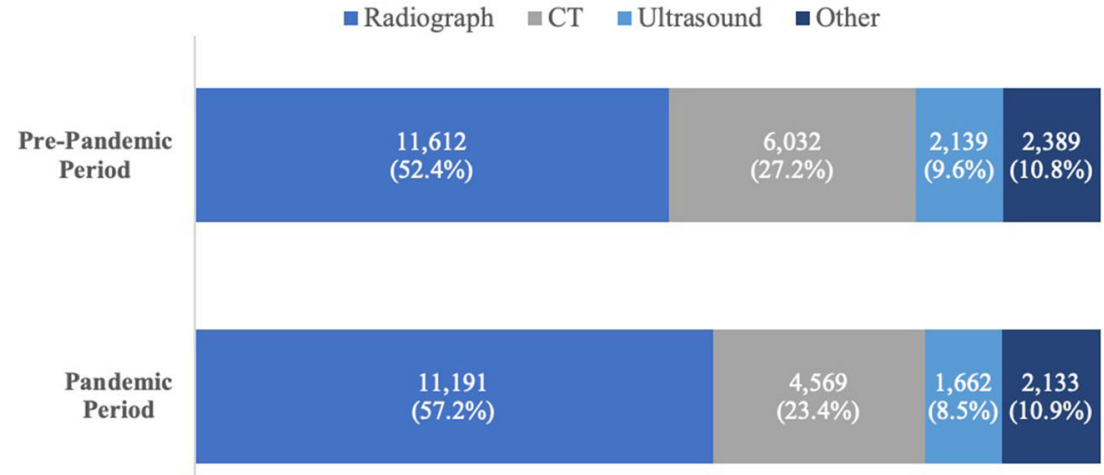

to either an inpatient or critical care unit. Moreover, patients with COVID-19 were imaged at a per-encounter rate (mean 4.0 exams) significantly greater than PUIs (2.8 exams) and those without COVID-19 (2.2 exams). The authors note that scarcity of COVID-19 tests may have led to rationing of such testing for the sickest patients, and such actions could result in bias. An analysis of imaging reports of all ED patients revealed that a greater proportion of patients during the pandemic had imaging reports with impressions that did not contain the phrases "no acute" or the word "normal" than during the pre-pandemic period; we postulate that this implies a greater percent of positive imaging during the pandemic period. In the aggregate, ED patient volumes were markedly reduced during the pandemic, but a slight increase in admission rate and increase in the percent of patients arriving by ambulance suggest there was a higher concentration of sicker patients who presented to the ED. Our data also suggest that patients with COVID-19 required greater imaging and other hospital resources due to the severity of their illness. While the observation that a greater proportion of ED patients arrived by ambulance as opposed to private vehicle may be interpreted to mean that these patients were more ill, it is also possible that the stay-at-home order in effect could have been interpreted by some to extend to private vehicle use, even if they needed emergent care, for which they may have viewed transportation by ambulance as the more appropriate option.

Notable changes in imaging ordering practices were observed in our study. In concert with a significant reduction in ED patient visits when compared to the pre-pandemic era, there was a significant decline $(12 \%)$ in the number of examinations that were ordered. A recent study that analyzed imaging output from five hospitals in the University of California system reported a $35 \%$ reduction in ED imaging [20]. While both studies observed significant reductions in ED imaging, the more drastic decline in the Houshyar et al. study is multifactorial and likely relates to regional differences in disease prevalence, magnitude and scope of social distancing mandates, variable adoption of these measures by members of the public, and existing image ordering practices and resources [20]. Within our system, despite a total reduction in ED imaging, proportional radiograph utilization increased from $52.4 \%$ of all imaging to $57.2 \%$, accounted for by a disproportionate increase in chest and thoracoabdominal radiography and relative decrease in axial and appendicular radiographs. A relative increase in the use of chest and thoracoabdominal radiography may be explained by the respiratory and gastrointestinal presentations of COVID-19, whereas the lockdown may have resulted in decreased ED visits for minor injuries and subsequent ordering of axial and appendicular radiographs [21]. Moreover, we also noted that, while the total number of CTs decreased during the pandemic period, the proportion of chest CT increased in patients diagnosed with or suspected to have COVID-19, a phenomenon likewise explained by the typical respiratory presentation of COVID-19 in addition to its documented association with pulmonary embolism and deep venous thrombosis [22]. A several-minutes cleaning protocol occurred between COVID-19+ or PUI patients who underwent CT; although it is doubtful that this resulted in significant a significant CT capacity impact, it is likely that infection prevention considerations for technologists, radiographers, and sonographers decreased non-essential imaging.

With respect to ultrasound, there was an overall significant reduction in utilization, accounted for by a marked decrease in non-COVID-19-related imaging; for example, only 4 pelvic ultrasound studies were performed in the 5371 COVID-19 patients or PUIs, which, at least in part, likely reflects efforts to protect sonographers from COVID-19 exposure for nonessential imaging. The proportion of upper and lower extremity and retroperitoneal (kidney, ureters, and urinary bladder) ultrasound examinations increased. As with the increased proportion of chest $\mathrm{CT}$, we postulate that an increase in extremity ultrasound related to the association between COVID-19 and venous thromboembolism. The increase in retroperitoneal ultrasound may be explained, in part, by emerging evidence at the time of a relationship between COVID-19 and acute renal injury [23]. However, we note that our numbers were small, with only 16 retroperitoneal ultrasound exams being done on 
COVID-19 patients. Furthermore, there were anecdotal reports within the local EDs of an association between COVID-19 and contrast-induced nephropathy. Though this link has not been documented in the literature, ED providers may have relied on retroperitoneal ultrasound to evaluate for renal pathology on the basis of suspicious drops in glomerular filtration rate in COVID-19 patients who had recently undergone contrast-enhanced CT.

\section{Limitations}

This study was not inclusive of all local hospitals as it only included data from four hospitals in a major metropolitan area. Additionally, as with any observational study, because of its non-randomized nature, this study may be subject to residual confounding. The effect of the pandemic may be directly related to the concentration of illness in a community and, as such, our findings may not be generalizable to communities which had significantly greater, or fewer, cases of COVID- 19 . The variable availability of COVID-19 testing capacity and clinical parameters used to order COVID-19 testing during the study period may have resulted in an underestimation of COVID-19 patients, with some of these patients being incorrectly classified as PUIs. In combination, the issues of testing scarcity, use of COVID-19 testing preferentially on the sickest patients, and the disease prevalence in the local population can lead to bias in our categorization of patients (COVID-19+, PUI, non-COVID-19), which may influence our results.

\section{Conclusions}

Presumably as a result of mandatory social distancing measures, the COVID-19 pandemic resulted in unique changes in several characteristics of both ED visits and imaging originating from the ED. This retrospective analysis shows that there was a $43.2 \%$ decrease in ED visits which occurred during the COVID-19 pandemic. ED visits were on average shorter, and patients were more likely to arrive by ambulance and more likely to be admitted. Overall, more radiographs but fewer CT and US were ordered during the pandemic period. But, ED patients with COVID-19 were imaged at a per-encounter rate (mean 4.0 exams) significantly greater than PUIs ( 2.8 exams) and those without COVID-19 (2.2 exams). There were also shifts in gender and racial characteristics of ED visits during the pandemic, with COVID-19 patients more likely to be male, Black, and older than in the comparison year. This study supports other reports of racial, age, and gender disparities in the COVID-19 pandemic.

Authors' contributions The authors confirm contribution to the paper as follows: study conception and design: TNH, JOJ, and CS; data analysis: $\mathrm{CS}$; interpretation of results: CS, TNH, KDH, MEZ, RLG, and JOJ; draft manuscript preparation: CS, TNH, KDH, MEZ, RLG, and JOJ. All the authors reviewed the results and approved the final version of the manuscript.

\section{Compliance with ethical standards}

Disclaimer The authors declare that they had full access to all of the data in this study and the authors take complete responsibility for the integrity of the data and the accuracy of the data analysis.

Conflict of interest The authors declare that they have no conflict of interest.

Ethics approval This study was reviewed and approved by our institutional IRB.

Consent to participate N/A

Consent for publication N/A

Code availability Analysis was performed using SAS 9.4.

\section{References}

1. Derraz I (2020) Stroke health care use and COVID-19. American Journal of Neuroradiology. 41:E36. https://doi.org/10.3174/ajnr. A6563

2. Stiepan D (2020) When to seek emergency care during COVID-19 pandemic. Mayo Clinic. https://newsnetwork.mayoclinic.org/ discussion/when-to-seek-emergency-care-during-covid-19pandemic/

3. Centers of Disease Control and Prevention (2020) Coronavirus disease 2019 (COVID-19)- what to do if you are sick, Centers of Disease Control and Prevention, Editor

4. Gutierrez L (2020) 'Heart attacks at home'? Amid COVID-19, people avoid the ER, and doctors are worried. https://www. kansascity.com/news/coronavirus/article242480521.html

5. McFarling U 'Where are all our patients?': COVID phobia is keeping people with serious heart symptoms away from ERs. STAT. https://www.statnews.com/2020/04/23/coronavirus-phobiakeeping-heart-patients-away-from-er/

6. Metzler B, Siostrzonek P, Binder RK, Bauer A, Reinstadler SJ (2020) Decline of acute coronary syndrome admissions in Austria since the outbreak of COVID-19: the pandemic response causes cardiac collateral damage. Eur Heart J 41(19):1852-1853. https:// doi.org/10.1093/eurheartj/ehaa314

7. Naccarato M, Scali I, Olivo S, Ajčević M, Buoite Stella A, Furlanis G, Lugnan C, Caruso P, Peratoner A, Cominotto F, Manganotti P (2020) Has COVID-19 played an unexpected "stroke" on the chain of survival? J Neurol Sci 414:116889. https://doi.org/10.1016/j.jns. 2020.116889

8. Wan Y-L, Schoepf UJ, Wu CC et al (9000) Preparedness and best practice in radiology department for COVID-19 and other future pandemics of severe acute respiratory infection. J Thorac Imaging, Publish Ahead of Print. https://doi.org/10.1097/rti. 0000000000000529

9. Hartnett KP, Kite-Powell A, DeVies J et al (2020) Impact of the COVID-19 pandemic on emergency department visits - United States, January 1, 2019-May 30, 2020. MMWR Morb Mortal Wkly Rep 69(23):699-704. https://doi.org/10.15585/mmwr. mm6923e1 
10. Oxley TJ, Mocco J, Majidi S, Kellner CP, Shoirah H, Singh IP, de Leacy RA, Shigematsu T, Ladner TR, Yaeger KA, Skliut M, Weinberger J, Dangayach NS, Bederson JB, Tuhrim S, Fifi JT (2020) Large-vessel stroke as a presenting feature of COVID-19 in the young. N Engl J Med 382(20):e60. https://doi.org/10.1056/ NEJMc2009787

11. Kim HS, Cruz DS, Conrardy MJ, Gandhi KR, Seltzer JA, Loftus TM, Fant AL, McCarthy DM (2020) Emergency department visits for serious diagnoses during the COVID-19 pandemic. Acad Emerg Med. 27:910-913. https://doi.org/10.1111/acem.14099

12. Lange, S. J., Ritchey, M. D., Goodman, A. B., et al. (2020). Potential indirect effects of the COVID-19 pandemic on use of emergency departments for acute life-threatening conditions United States, January-May 2020. MMWR Morb Mortal Wkly Rep, 69(25), 795-800. 10.15585/mmwr.mm6925e2

13. Georgia Executive Order. 03.23.20.01

14. City of Atlanta. Executive Order Number 2020-21

15. Georgia Executive Order. 04.27.20.01

16. Lerner EB, Newgard CD, Mann NC (2020) Effect of the coronavirus disease 2019 (COVID-19) pandemic on the U.S. Emergency Medical Services System: A Preliminary Report. Acad Emerg Med. https://doi.org/10.1111/acem.14051

17. Garcia S, Albaghdadi MS, Meraj PM, Schmidt C, Garberich R, Jaffer FA, Dixon S, Rade JJ, Tannenbaum M, Chambers J, Huang PP, Henry TD (2020) Reduction in ST-Segment elevation cardiac catheterization laboratory activations in the United States during COVID-19 pandemic. J Am Coll Cardiol. 75:2871-2872. https://doi.org/10.1016/j.jacc.2020.04.011
18. Zhao J, Li H, Kung D, Fisher M, Shen Y, Liu R (2020) Impact of the COVID-19 epidemic on stroke care and potential solutions. Stroke 51(7):1996-2001. https://doi.org/10.1161/STROKEAHA. 120.030225

19. Bambra C, Riordan R, Ford J, Matthews F (2020) The COVID-19 pandemic and health inequalities. J Epidemiol Community Health. https://doi.org/10.1136/jech-2020-214401

20. Houshyar R, Tran-Harding K, Glavis-Bloom J, Nguyentat M, Mongan J, Chahine C, Loehfelm TW, Kohli MD, Zaragoza EJ, Murphy PM, Kampalath R (2020) Effect of shelter-in-place on emergency department radiology volumes during the COVID-19 pandemic. Emerg Radiol. 27:781-784. https://doi.org/10.1007/ s10140-020-01797-y

21. Pascarella G, Strumia A, Piliego C, Bruno F, del Buono R, Costa F, Scarlata S, Agrò FE (2020) COVID-19 diagnosis and management: a comprehensive review. J Intern Med 288(2):192-206. https://doi. org/10.1111/joim.13091

22. Di Minno A, Ambrosino P, Calcaterra I et al (2020) COVID-19 and venous thromboembolism: a meta-analysis of literature studies. Semin Thromb Hemost. 46:763-771. https://doi.org/10.1055/s0040-1715456

23. Ronco C, Reis T, Husain-Syed F (2020) Management of acute kidney injury in patients with COVID-19. Lancet Respir Med 8(7):738-742. https://doi.org/10.1016/S2213-2600(20)30229-0

Publisher's note Springer Nature remains neutral with regard to jurisdictional claims in published maps and institutional affiliations. 